\title{
Quantum Backflow States from Eigenstates of the Regularized Current Operator
}

\author{
J.J.Halliwell.* E.Gillman, O.Lennon, M.Patel, and I.Ramirez \\ Blackett Laboratory \\ Imperial College \\ London $S W 72 B Z$ \\ $U K$
}

\begin{abstract}
We present an exhaustive class of states with quantum backflow - the phenomenon in which a state consisting entirely of positive momenta may have negative current and the probability flows in the opposite direction to the momentum. They are characterized by a general function of momenta subject to very weak conditions. Such a family of states is of interest in the light of a recent experimental proposal to measure backflow. We find one particularly simple state which has surprisingly large backflow - about 41 percent of the lower bound on flux derived by Bracken and Melloy. We study the eigenstates of a regularized current operator and we show how some of these states, in a certain limit, lead to our class of backflow states. This limit also clarifies the correspondence between the spectrum of the regularized current operator, which has just two non-zero eigenvalues in our chosen regularization, and the usual current operator.
\end{abstract}

PACS numbers: 03.65.Xp, 03.65.Yz., 03.65.Ta

*Electronic address: j.halliwell@imperial.ac.uk 


\section{INTRODUCTION}

The backflow effect is the intriguing quantum-mechanical phenomenon in which the current at the origin can be negative for a particle described by a wave function consisting entirely of positive momenta. This means that the probability of remaining in $x<0$ may, for certain states, increase with time, even though the momenta point out of the region.

This non-classical effect was first noted by Allcock [1] and subsequently explored in detail by Bracken and Melloy [2 4]. The existence of the effect is often noted in connection with the arrival time problem [1, 5-13]. More recently there have been a number of papers on

backflow [14 19] including an interesting proposal to measure it experimentally [20]. All of these recent papers give examples of states exhibiting backflow but to date there has been no systematic approach to finding such states. The purpose of this paper is to present an essentially exhaustive class of states with backflow, which in momentum space, have the general form

$$
\phi(p)=N \theta(p)(a-p) f(p)
$$

where $f(p)$ is a general complex function of momentum subject only to some simple restrictions involving the complex constant $a$ and the low moments of $f(p)$. In particular, we find that for any $f(p)$ for which the current exists and is non-zero, there are always some values of $a$ for which these states are backflow states. We then show how this set of states naturally appears from a study of the spectrum of a regularized current operator. We also clarify the correspondence between the spectrum of the regularized current, which has some unusual features, and the usual current. The class of states Eq.(1.1) may be of value in experimental measurements of backflow, since it is clearly of value to possess the largest possible set of possible states exhibiting backflow.

We describe the current and its properties in Section 2 and give examples of backflow states. In Section 3 we discuss the states of the form Eq.(1.1) and derive the conditions under which they give backflow. In Section 4 we consider the case where $f(p)$ is gaussian and compute its flux during the time interval where the current is negative, which turns out to be surprisingly large for such a simple state.

In Section 5 we introduce a regularization of the current operator and find its eigenstates. These states do not in general have negative (unregularized) current, so in Section 6 we show how, by taking a certain carefully chosen limit, we naturally generate backflow states of the 
form Eq.(1.1). We summarize and conclude in Section 7.

\section{THE CURRENT AND ITS PROPERTIES}

We begin by reviewing the properties of the current. We consider a free particle with initial wave function $\psi(x)$ centred in $x<0$ and consisting entirely of positive momenta. The current arises when we consider the amount of probability flux $F\left(t_{1}, t_{2}\right)$ crossing the origin during the time interval $\left[t_{1}, t_{2}\right]$, defined as a difference of two probabilities,

$$
\begin{aligned}
F\left(t_{1}, t_{2}\right) & =\int_{-\infty}^{0} d x\left|\psi\left(x, t_{1}\right)\right|^{2}-\int_{-\infty}^{0} d x\left|\psi\left(x, t_{2}\right)\right|^{2} \\
& =\int_{t_{1}}^{t_{2}} d t J(t)
\end{aligned}
$$

where $J(t)$ is the quantum-mechanical current at the origin

$$
J(t)=-\frac{i \hbar}{2 m}\left(\psi^{*}(0, t) \frac{\partial \psi(0, t)}{\partial x}-\frac{\partial \psi^{*}(0, t)}{\partial x} \psi(0, t)\right)
$$

The flux is also easily rewritten in terms of the Wigner function [21] at time t, $W_{t}(p, q)$,

$$
F\left(t_{1}, t_{2}\right)=\int_{t_{1}}^{t_{2}} d t \int d p d q \frac{p}{m} \delta(q) W_{t}(p, q)
$$

From this we see that the flux and current are classically positive for states with positive momenta, but can be negative in the quantum case, since the Wigner function can be negative. This is backflow.

The current is conveniently written in terms of the current operator

$$
\hat{J}=\frac{1}{2 m}(\hat{p} \delta(\hat{x})+\delta(\hat{x}) \hat{p})
$$

and the current in a state $|\psi\rangle$ then is

$$
J(t)=\langle\psi|\hat{J}(t)| \psi\rangle
$$

Similarly, the flux may be written

$$
F\left(t_{1}, t_{2}\right)=\left\langle\psi\left|\hat{F}\left(t_{1}, t_{2}\right)\right| \psi\right\rangle
$$

where

$$
\hat{F}\left(t_{1}, t_{2}\right)=\int_{t_{1}}^{t_{2}} d t \hat{J}(t)
$$


Some useful results on various aspects of the current may be found in Refs. [22 26]. Here we concentrate on some results specifically relating to backflow. Although the current can be arbitrarily negative there are restrictions on both the temporal and spatial extent of backflow and these both give useful measures of the amount of backflow for a given state. By considering the spectrum of the flux operator restricted to positive momenta, Bracken and Melloy have shown that the temporal extent of backflow is limited by a lower bound on the flux,

$$
F\left(t_{1}, t_{2}\right) \geq-c_{b m}
$$

where, interestingly, $c_{b m}$ is a a pure number independent of $\hbar$, the mass $m$ and the time interval [2]. It was computed numerically and found to be

$$
c_{b m} \approx 0.038452
$$

This means that the usually decreasing probability of remaining in $x<0$ can increase by no more than about 4 percent. This computation was repeated by Penz et al, who determined numerically the form of the maximizing backflow state [15], and also by Eveson et al [14].

The limitation on the spatial extent of backflow is indicated by a theorem of Eveson et al [14] who showed that the current at point $x, J(x)$, for states of positive momenta, satisfies

$$
\int d x J(x)|g(x)|^{2} \geq-\frac{\hbar}{8 \pi m} \int d x\left|\frac{d g}{d x}\right|^{2}
$$

for some smearing function $g(x)$. For example, for the particular case of a gaussian smearing,

$$
|g(x)|^{2}=\frac{1}{\sqrt{2 \pi \sigma^{2}}} \exp \left(-\frac{x^{2}}{2 \sigma^{2}}\right)
$$

we have

$$
\int d x J(x)|g(x)|^{2} \geq-\frac{\hbar}{32 \pi m \sigma^{2}}
$$

This can be rewritten in the suggestive form

$$
\frac{m \sigma^{2}}{\hbar} J\left(x_{0}\right) \geq-\frac{1}{32 \pi}
$$

where $x_{0}$ is a spatial point within the smearing region. The quantity $m \sigma^{2} / \hbar$ is the timescale for a wave packet to spread a distance $\sigma$, so this form indicates that the current can be extremely negative in a region of size $\sigma$ as long as the spreading time over that region is very short. Both the inequalities Eqs.(2.10), (2.13) indicate useful measures of the amount 
of backflow. Only Eq.(2.10) has been used in this way to date. It would be useful to explore Eq.(2.13) in a similar vein, e.g., to find out what sort of states are close to equality. Another measure of backflow, essentially the fraction of the $x$-axis on which the current is negative, was given by Berry [17].

We now give some specific examples of backflow states. The simplest example is a superposition of plane waves, given by Bracken and Melloy [2]. The experiment proposed in Ref.[20] involves a state of this general type. A generalization of this is the state given by Berry, of the form $(1-a \exp (i x))^{N}$, which has large negative current for large $N$ [17]. However, these states are not normalizable. Superpositions of gaussians were considered in Ref.[19], which are normalizable, but have a small tail of negative momentum, so one has to estimate how much of the backflow comes from this. A better example, truncated gaussian restricted to $p>0$, was given by Muga et al [16]. Two more examples are the state

$$
\phi(p)=\frac{18}{\sqrt{35 K}} \theta(p) p\left(e^{-p / K}-\frac{1}{6} e^{-p / 2 K}\right)
$$

given by Bracken and Melloy [2] and the state

$$
\phi(p)=N \theta(p) \theta\left(p_{0}-p\right)\left(p \sqrt{3}-p_{0}\right)
$$

given by Eveson et al [14]. All of the above states, when normalizable, have small backflow, just a few percent of the maximum flux. Yearsley et al [19] undertook a search for an analytic expression matching the optimal backflow state obtained numerically by Penz et al [15]. They found two states constructed from Fresnel functions which gave good backflow, one of which was about 70 percent of the maximum possible. There are some indications that this result could be considerably improved on using Airy functions [27]. We also mention some interesting states written down by Strange in the context of three dimensional systems, where the periodicity of the coordinates allows for backflow states that can persist indefinitely in time [18].

Most of the above states come from simple guesswork and it would be desirable to have a general and systematic picture of what a backflow state looks like. This we do in the following sections.

Finally, we illustrate backflow in Fig.(1), where we plot the probability of remaining in $x<0$ for the maximal backflow state obtained numerically by Penz et al [15]. It decreases on the whole, but may increase for periods of time, a classically unexpected result. 


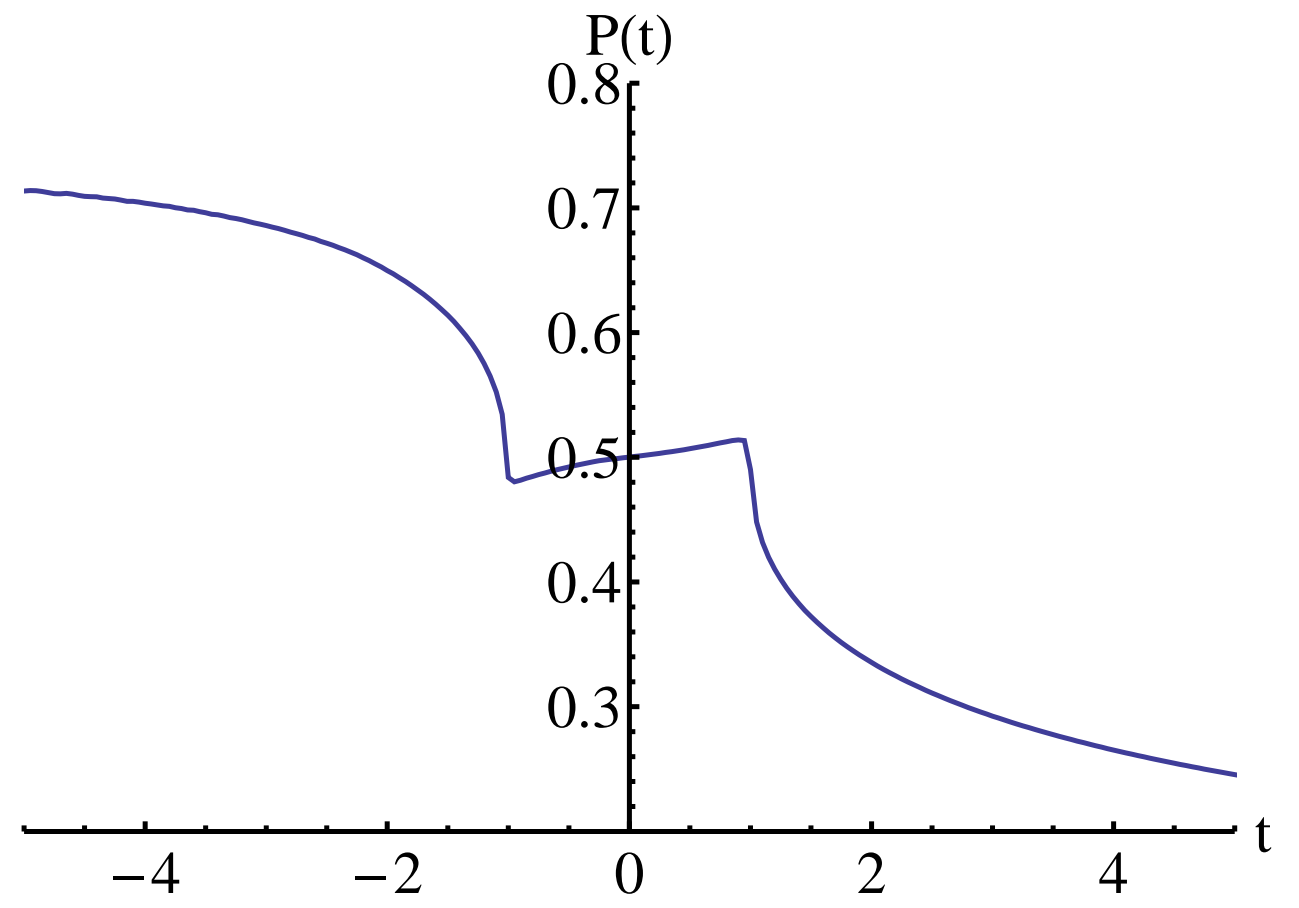

FIG. 1: The probability $P(t)$ that the state will be found in $x<0$ at time $t$ for a wave function with backflow.

\section{A LARGE FAMILY OF BACKFLOW STATES}

We now exhibit an exhaustive family of states with backflow. In terms of the momentum space wave functions $\phi(p)$ the current is

$$
J=\frac{1}{4 \pi m \hbar}\left(u v^{*}+u^{*} v\right)
$$

where

$$
\begin{aligned}
& u=\int d p \phi(p) \\
& v=\int d p p \phi(p)
\end{aligned}
$$

This means that $\phi(p)$ must fall off faster than $1 / p^{2}$ for large $p$ for the current to exist. Backflow states are states of positive momenta with

$$
u v^{*}+u^{*} v<0
$$


We have found that a particularly convenient way to solve this inequality is to write the state in the form

$$
\phi(p)=N \theta(p)(a-p) f(p)
$$

where $N$ is real and $a$ is complex and $f(p)$ is a general complex function which, for convenience, we normalize according to

$$
\int_{0}^{\infty} d p|f(p)|^{2}=1
$$

and which must fall off faster than $1 / p^{3}$ for $\phi(p)$ to have the right behaviour. Clearly any state $\phi(p)$ can be written in this form, subject to the above fall off conditions, so the form Eq.(3.4) is completely general.

The current of the states Eq.(3.4) is negative under the condition

$$
\operatorname{Re}\left(\left(a f_{0}-f_{1}\right)\left(a f_{1}^{*}-f_{2}^{*}\right)\right)<0
$$

where we have introduced the three quantities

$$
f_{n}=\left\langle 0\left|\hat{p}^{n}\right| f\right\rangle=\frac{1}{\sqrt{2 \pi \hbar}} \int_{0}^{\infty} d p p^{n} f(p)
$$

for $n=0,1,2$. These quantities all exist since $f(p)$ falls off fast enough for large $p$. Eq.(3.6) is a condition on the complex constant $a$ for given $f_{n}$ and reads

$$
\left(f_{0} f_{1}^{*}+f_{0}^{*} f_{1}\right)|a|^{2}-\left(f_{0} f_{2}^{*}+\left|f_{1}\right|^{2}\right) a-\left(f_{0}^{*} f_{2}+\left|f_{1}\right|^{2}\right) a^{*}+f_{1} f_{2}^{*}+f_{1}^{*} f_{2}<0
$$

We show that this inequality is always satisfied for some $a$.

First we deal with the simple cases. If $\left(f_{0} f_{1}^{*}+f_{0}^{*} f_{1}\right)<0$, then Eq.(3.8) is clearly satisfied for sufficiently large $a$ (and the current can be arbitrarily negative in this case). If $f_{1}=0$, it is satisfied for $\left(f_{0} f_{2}^{*}+f_{0}^{*} f_{2}\right) a>0$. If $f_{0}=0$, it is satisfied for $a>\left(f_{1} f_{2}^{*}+f_{1}^{*} f_{2}\right) /\left(2\left|f_{1}\right|^{2}\right)$. If both $f_{0}$ and $f_{2}$ both vanish, it is satisfied for all $a$ unless $f_{1}=0$ in which case $J=0$. Similarly, the cases $f_{0}=0=f_{1}$ and $f_{1}=0=f_{2}$ both imply that $J=0$.

The only non-trivial case is the case $\left(f_{0} f_{1}^{*}+f_{0}^{*} f_{1}\right)>0$ and in this case we write Eq.(3.8) as

$$
A|a|^{2}-B a-B^{*} a^{*}+C<0
$$

where the coefficients $A, B, C$ are easily read off and we have $A>0$. This may be rewritten

$$
A^{2}\left|a-\frac{B^{*}}{A}\right|^{2}-|B|^{2}+A C<0
$$


Now note that

$$
\begin{aligned}
|B|^{2}-A C & =\left.\left.\left|f_{0} f_{2}^{*}+\right| f_{1}\right|^{2}\right|^{2}-\left(f_{0} f_{1}^{*}+f_{0}^{*} f_{1}\right)\left(f_{1} f_{2}^{*}+f_{1}^{*} f_{2}\right) \\
& =\left|f_{1}^{2}-f_{0} f_{2}\right|^{2}>0
\end{aligned}
$$

This means that the inequality Eq.(3.10) is always satisfied if we choose $a$ to be sufficiently close to the value $a=B^{*} / A$. (This value also gives the most negative possible current in the case $A>0)$.

We therefore see that as long as the current exists and is non-zero, there is always some value of the complex constant $a$ which ensures that states of the form Eq.(3.4) have negative current. This is our first main result.

Almost all of the backflow states written down to date involve real $f(p)$ and $a$, so we write out the conditions more explicitly for this case. When $f_{1} f_{0}<0$, this means that $a$ must satisfy $a>f_{2} / f_{1}$. When $f_{1} f_{0}>0$, we require

$$
\min \left\{\frac{f_{1}}{f_{0}}, \frac{f_{2}}{f_{1}}\right\}<a<\max \left\{\frac{f_{1}}{f_{0}}, \frac{f_{2}}{f_{1}}\right\}
$$

If $f_{1}=0$ the condition is only satisfied if $a f_{0} f_{2}>0$. If $f_{0}=0$, we require $a>f_{2} / f_{1}$.

It is easy to show that the above conditions are satisfied for some of the specific backflow states given earlier, such as Eqs.(2.15), (2.16), for suitable choice of $a$. Furthermore, because the form Eq.(3.4) is general all other backflow states must be expressable in this form, for suitable choices of $a$ and $f(p)$ satisfying the above conditions.

\section{A SIMPLE STATE WITH SUBSTANTIAL BACKFLOW}

We now give a simple example of a state of the type Eq.(3.4) which has significant negative flux. We choose $f(p)$ to be a simple gaussian and thus the state is

$$
\phi(p)=N(a-p) e^{-\gamma_{0}^{2} p^{2}}
$$

where $a$ and $\gamma_{0}$ are real constants to be determined and the normalization factor $N$ is given by

$$
N^{2}=2 \sqrt{\frac{2}{\pi}} \gamma_{0}\left(a^{2}+\frac{1}{4 \gamma_{0}^{2}}-\sqrt{\frac{2}{\pi}} \frac{a}{\gamma_{0}}\right)^{-1}
$$


This simple state has the advantage that its current at any time may be calculated analytically and is

$$
J(t)=\frac{N^{2}}{32 \pi m \hbar|\gamma(t)|^{6}} \gamma^{*}(t)\left[a \gamma^{*}(t) \sqrt{\pi}-1\right][2 a \gamma(t)-\sqrt{\pi}]+c . c .
$$

where $\gamma(t)=\left(\gamma_{0}^{2}+i t / 2 m \hbar\right)^{\frac{1}{2}}$. It depends mainly on the dimensionless quantity $a \gamma_{0}$ and at $t=0$ is clearly negative when

$$
\frac{1}{\sqrt{\pi}}<a \gamma_{0}<\frac{\sqrt{\pi}}{2}
$$

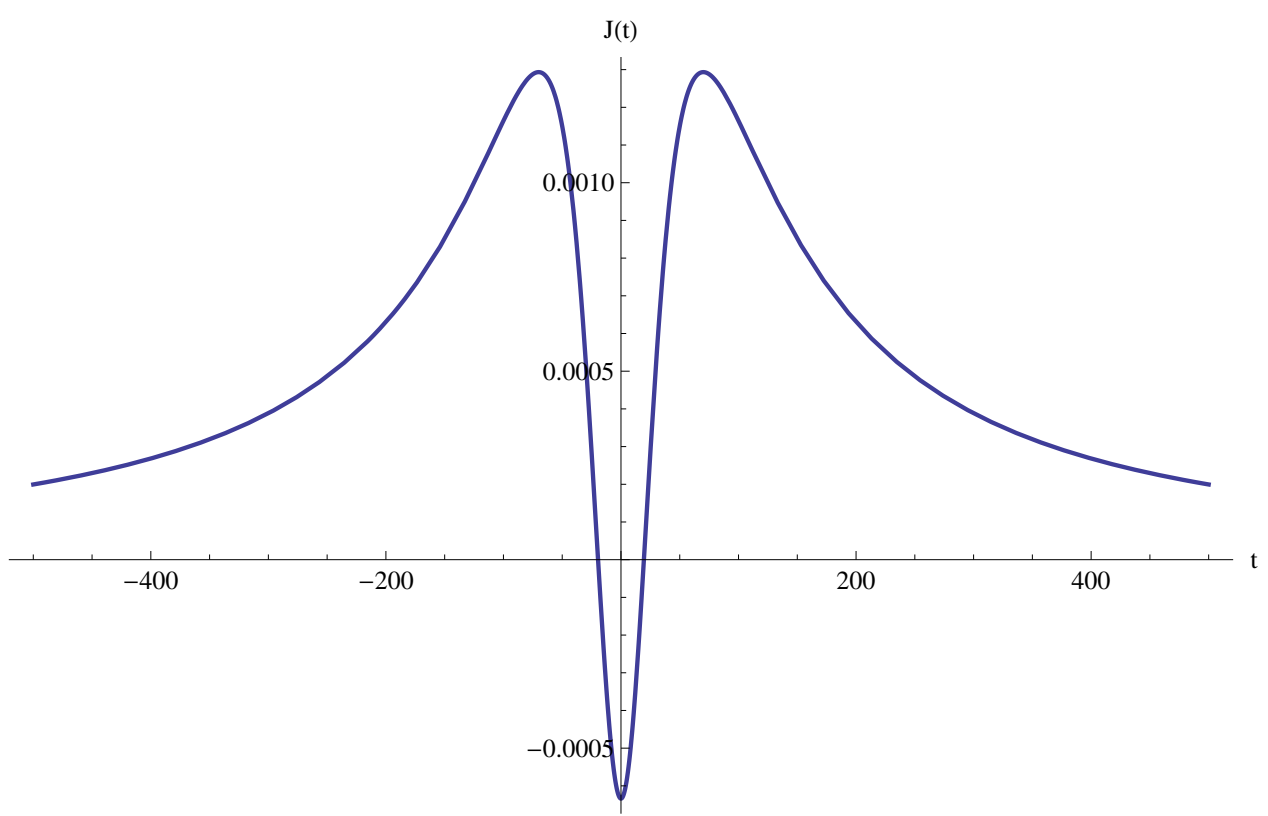

FIG. 2: The current as a function of time $J(t)$ for the state Eq.(4.1). It has a clear period of backflow around $t=0$.

The current is plotted in Fig.(2) and clearly has substantial backflow around $t=0$, where the parameter $a \gamma_{0}$ has been adjusted numerically to give the most negative flux, which takes place at $a \gamma_{0} \approx 0.684$. The flux in the time interval where the current is negative may be computed by numerical integration and we find the result

$$
F\left(t_{1}, t_{2}\right) \approx-0.01573
$$

which is about 41 percent of the Bracken-Melloy bound $c_{b m}$. One can also check the normalization by integrating over a large range of times numerically and we find that the total 
flux is 1 , as expected. We have also explored complex values of the constant $a$ but this leads to smaller negative flux.

This value for the flux is on the one hand rather far from the maximum backflow. On the other hand, it is surprisingly large for such a simple state - most of the known backflow states discussed in Section 2 have a flux which is only a few percent of the Bracken-Melloy bound. States of the general form Eq.(4.1) are not difficult to produce experimentally although the hard part is clearly the restriction to $p>0$. Nevertheless, the surprisingly large backflow manifested by such a simple state may be useful in experimental measurement of backflow.

\section{THE REGULARIZED CURRENT AND ITS EIGENSTATES}

We now consider how backflow states of the form Eq.(3.4) might arise from a study of current operator. Since backflow states are states of positive momenta with $\langle\psi|\hat{J}| \psi\rangle<0$ one might be tempted to find them by considering the eigenstates of the current operator $\hat{J}$. However, because of the presence of the $\delta$-function in $\hat{J}$ this operator is poorly defined and its eigenstates do not exist. To go further down this route, therefore, it is necessary to regularize the current operator somehow. This then leaves the question of how to relate this to the average of the unregularized current operator, but this will be addressed below.

We focus on a regularized current operator of the form

$$
\hat{J}_{r e g}=\frac{1}{2 m}\left(\hat{p} \delta_{\sigma}(\hat{x})+\delta_{\sigma}(\hat{x}) \hat{p}\right)
$$

where $\delta_{\sigma}(\hat{x})$ is a regularization of the $\delta$-function. Since we are interested in positive momentum states only we focus on the eigenstates of the operator $\hat{J}_{\text {reg }}^{p>0}=\theta(\hat{p}) \hat{J}_{\text {reg }} \theta(\hat{p})$.

Numerous regularizations of the current operator have been proposed [19, 26, 28, 29], some of which are related to specific experimental procedures. However, we have found only one in which the restriction to positive momentum is easily implemented, due to Mason et al [26], which we follow (although note that what we do below is different, since Mason et al were not interested in the positive momentum sector). We first note that the $\delta$-function operator may be written

$$
\delta(\hat{x})=|0\rangle\langle 0|
$$

where $|0\rangle$ denotes the position eigenstate $|x\rangle$ at $x=0$. We can regularize this as

$$
\delta_{\sigma}(\hat{x})=\frac{1}{\sigma}\left|f_{\sigma}\right\rangle\left\langle f_{\sigma}\right|
$$


by finding a family of states $\left|f_{\sigma}\right\rangle$, which are normalized $\left\langle f_{\sigma} \mid f_{\sigma}\right\rangle=1$ and such that

$$
\frac{\left|f_{\sigma}\right\rangle}{\sigma^{\frac{1}{2}}} \rightarrow|0\rangle
$$

as $\sigma \rightarrow 0$. An example of such a state is the gaussian

$$
f_{\sigma}(p)=\sqrt{\frac{\sigma}{2 \pi \hbar}} \exp \left(-\frac{\sigma^{2} p^{2}}{\alpha^{2} \hbar^{2}}\right)
$$

where $\alpha^{2}=32 \pi$ to give the correct normalization (over an infinite range of $p$ ). Our regularized current operator on positive momentum is then

$$
\hat{J}_{\text {reg }}^{p>0}=\frac{1}{2 m \sigma} \theta(\hat{p})(\hat{p}|f\rangle\langle f|+| f\rangle\langle f| \hat{p}) \theta(\hat{p})
$$

where for notational simplicity we drop the $\sigma$-dependence in $|f\rangle$. We now note that the $\theta$-functions may be absorbed into the definition of $|f\rangle$, so hereafter we assume that $|f\rangle$ are states of purely positive momenta. (Note this implies a different value of $\alpha$ in the above gaussian, although this is not important in what follows). Since we are only concerned with the positive momentum regime, in what follows we also for notational simplicity denote the regularized current on positive momentum simply by $\hat{J}_{\text {reg }}$.

The spectrum of $\hat{J}_{\text {reg }}$ is determined very easily. There are just two non-zero eigenvalues, one positive, one negative,

$$
\lambda_{ \pm}=\frac{1}{2 m \sigma}\left( \pm\left\langle\hat{p}^{2}\right\rangle_{f}^{\frac{1}{2}}+\langle\hat{p}\rangle_{f}\right)
$$

and the eigenstates are

$$
\left|\phi_{ \pm}\right\rangle=N\left(\left\langle\hat{p}^{2}\right\rangle_{f}^{\frac{1}{2}} \pm \hat{p}\right)|f\rangle
$$

where $\left\langle\hat{p}^{2}\right\rangle_{f}=\left\langle f\left|\hat{p}^{2}\right| f\right\rangle$, and similarly for $\langle\hat{p}\rangle_{f}$ and $N$ is a normalization factor. There are also an infinite number of eigenstates with eigenvalue zero but these are not relevant to our considerations. Some (but not all) other regularizations give eigenvalues with the same general features [26, 28, 29] - just two non-zero eigenvalues, one positive, one negative. This somewhat unusual feature, which means that there is only one independent state for which $\left\langle\hat{J}_{\text {reg }}\right\rangle<0$, does not immediately reconcile with the fact that the usual current has infinitely many states for which $\langle\hat{J}\rangle<0$, but we will clarify this in the next section. 


\section{GENERATING BACKFLOW STATES FROM THE REGULARIZED CUR- RENT EIGENSTATES}

Consider now the relationship between the spectrum of the regularized current operator and the usual current and the question of whether we can use the negative eigenstates of $\hat{J}_{\text {reg }}$ to generate backflow states, states with $\langle\hat{J}\rangle<0$. In particular, we consider a limit of the form

$$
\langle\psi|\hat{J}| \psi\rangle=\lim _{\sigma \rightarrow 0}\left\langle\phi_{-}\left|\hat{J}_{\text {reg }}\right| \phi_{-}\right\rangle
$$

However, the limit $\sigma \rightarrow 0$ of the spectrum of the regularized current operator does not exist - the eigenvalues go to $\pm \infty$ and the eigenstates become ill-defined, so this does not generate states $|\psi\rangle$ with $J<0$.

One might instead choose a fixed $|f\rangle$ and keep the eigenstate $\left|\phi_{-}\right\rangle$fixed so take the limit in $\hat{J}_{\text {reg }}$ only, thereby computing the current of the state $\left|\phi_{-}\right\rangle$. However, there is no guarantee that this remains negative. The point here is that although the negative eigenstate $\left|\phi_{-}\right\rangle$is of the form of the family of backflow states Eq.(3.4) with $a=\left\langle\hat{p}^{2}\right\rangle_{f}^{\frac{1}{2}}$, the quantity $\left\langle\hat{p}^{2}\right\rangle_{f}$ is not simply related to the moments $f_{0}, f_{1}, f_{2}$ (which are moments of $\phi(p)$ not of $|\phi(p)|^{2}$ ), so there is no guarantee that the inequalities ensuring negative current are satisfied, and indeed they are not for the case of gaussian $f(p)$, as one can easily check. Hence a more subtle approach is required to extract backflow states from the regularized current eigenstate $\left|\phi_{-}\right\rangle$.

One can see that the problem in Eq.(6.1) is that as $\sigma \rightarrow 0$ in $\hat{J}_{\text {reg }}$, the state $\left|\phi_{-}\right\rangle$does not obviously remain in the negative part of the spectrum of $\hat{J}_{\text {reg }}$. This is further complicated by the fact that the spectral decomposition of $\hat{J}_{\text {reg }}$

$$
\hat{J}_{\text {reg }}=\lambda_{+}\left|\phi_{+}\right\rangle\left\langle\phi_{+}\left|+\lambda_{-}\right| \phi_{-}\right\rangle\left\langle\phi_{-}\right|
$$

becomes ill-defined in the limit. However, these observations give us a clue as to how to proceed. The key is to evolve the state also in the limiting procedure in Eq.(6.1), in such a way that the expression remains negative but the state remains well-defined. The state generated in this way will no longer be an eigenstate of a regularized current operator, but it will be a backflow state, which is what we seek.

To this end, we choose a fixed fiducial state $|f\rangle$ and a family of states $|g\rangle$ of positive momenta which will interpolate from the initial value $|g\rangle=|f\rangle$ to the limiting value 
$|g\rangle / \sigma^{\frac{1}{2}} \rightarrow|0\rangle$. We define a regularized current operator for $|g\rangle$,

$$
\hat{J}_{\text {reg }}(g)=\frac{1}{2 m \sigma}(\hat{p}|g\rangle\langle g|+| g\rangle\langle g| \hat{p})
$$

which therefore interpolates from the original regularized current operator $\hat{J}_{\text {reg }}=\hat{J}_{\text {reg }}(f)$ for fixed $f$ to the usual unregularized current operator $\hat{J}$. We also define a set of states

$$
\left|\psi_{a}\right\rangle=N\left(a_{f g}-\hat{p}\right)|f\rangle
$$

for some complex number $a_{f g}$ which depends on $|f\rangle$ and $|g\rangle$ and is such that $a_{f f}=\left\langle p^{2}\right\rangle_{f}^{\frac{1}{2}}$ when $|f\rangle=|g\rangle$, so it is initially an eigenstate of $\hat{J}_{\text {reg }}(f)$ with negative eigenvalue, but will evolve with $|g\rangle$. These states are of course clearly of the form Eq.(3.4).

The aim is now to evolve the quantity $\left\langle\psi_{a}\left|\hat{J}_{r e g}(g)\right| \psi_{a}\right\rangle$ from its initial negative value in such a way that it remains negative as $\hat{J}_{\text {reg }}$ approaches $\hat{J}$ thereby obtaining a backflow state. This can be achieved by evolving $a_{f g}$ in a suitable way. Although the resulting current operator obtained in this limit is singular the state remains well-defined (since only $a_{f g}$ changes), so the average of the current thereby obtained is well-defined. We have

$$
\left\langle\psi_{a}\left|\hat{J}_{\text {reg }}(g)\right| \psi_{a}\right\rangle=\frac{N^{2}}{4 m \sigma} \operatorname{Re}\left(\left\langle f\left|\left(a_{f g}^{*}-\hat{p}\right) \hat{p}\right| g\right\rangle\left\langle g\left|\left(a_{f g}-\hat{p}\right)\right| f\right\rangle\right)
$$

and this is negative for all $|g\rangle$ as long as $a_{f g}$ satisfies

$$
\operatorname{Re}\left(\left(a_{f g}^{*}\langle f|\hat{p}| g\rangle-\left\langle f\left|\hat{p}^{2}\right| g\right\rangle\right)\left(a_{f g}\langle g \mid f\rangle-\langle g|\hat{p}| f\rangle\right)\right)<0
$$

Now note that these expressions remain well-defined in the limit $|g\rangle / \sigma^{\frac{1}{2}} \rightarrow|0\rangle$ and the quantities of the form $\left\langle g\left|\hat{p}^{n}\right| f\right\rangle$ tend to the moments $f_{n}=\left\langle 0\left|\hat{p}^{2}\right| f\right\rangle$ (up to a factor of $\sigma^{\frac{1}{2}}$ ) defined in Eq.(3.7). We may therefore take this limit, so $a_{f g} \rightarrow a_{f 0}$ and the restriction on $a_{f 0}$ for negative current becomes

$$
\operatorname{Re}\left(\left(a_{f 0}^{*} f_{1}^{*}-f_{2}^{*}\right)\left(a_{f 0} f_{0}-f_{1}\right)\right)<0
$$

which is precisely the earlier condition Eq.(3.6) and Eq.(6.5) becomes the current Eq.(3.1).

We have thus shown that the negative eigenvalue eigenstate Eq.(15.8) of the regularized current operator, although not itself a backflow state, may be distorted into the general family of backflow states Eq.(3.4), by distorting the value of $a_{f g}$ in a suitable way. In this sense, the eigenstates of the regularized current operator may be used to generate the family of backflow states Eq.(3.4). 
This connection also shines some light on the unusual properties of the spectrum of $\hat{J}_{\text {reg }}$ noted above. Although it seems to have only one state with $\left\langle\hat{J}_{\text {reg }}\right\rangle<0$, there is one state for every regularization function $f$. There are of course an infinite number of such functions and infinite number of ways of regularizing the current. So our infinite family of backflow states corresponds to an infinite family of regularizations of the current operator.

Note also that although we introduced the family of states $|f\rangle$ as regularizations of the $\delta$-function, Eq.(5.3), there is in the end no requirement that these functions lead to Eq.(5.3) being "close" in any sense to the $\delta$-function $\delta(\hat{x})$. All that is required of these states is that the current exists and is non-zero in the states Eq.(3.4).

\section{SUMMARY AND CONCLUSIONS}

We have presented an exhaustive family of states Eq.(3.4) exhibiting backflow. They are characterized by a general function $f(p)$, subject to some simple restrictions on its first three moments and the constant $a$. In particular, for any complex function $f(p)$ which is such that the current exists and is non-zero, these states are always backflow states for some value of the complex constant $a$. Since any state may be expressed in the form Eq.(3.4), subject to the appropriate fall off conditions, this state is completely general so all backflow states must be expressable in this form and satisfy the conditions we derived. We also exhibited a very simple state with surprisingly large negative flux, which may be of experimental relevance.

We considered the spectrum of the regularized current operator, in a specific regularization. Negative eigenstates of this operator are not necessarily backflow states. However, we showed how to take a certain limit in which they became backflow states, and in particular, we generated the family Eq.(3.4), with exactly the same restrictions on $f(p)$. We also noted the correspondence between families of backflow states and families of regularizations of the current operator, which explains why it is consistent for each regularized current operator to have just one state with $\left\langle\hat{J}_{\text {reg }}\right\rangle<0$. 


\section{ACKNOWLEDGEMENTS}

We are very grateful to Gonzalo Muga and James Yearsley for useful discussions. JJH was supported by EPSRC grant EP/J008060/1.

[1] G.R.Allcock, Ann.Phys 53, 253 (1969); 53, 286 (1969); 53, 311 (1969).

[2] A.J.Bracken and G.F.Melloy, J.Phys. A27, 2197 (1994).

[3] A.J.Bracken and G.F.Melloy, Found.Phys. 28, 505 (1998).

[4] A.J.Bracken and G.F.Melloy, Ann.Phys.(Leipzig) 7, 726 (1998).

[5] W.R.McKinnon and C.R.Leavens, Phys.Rev. A51, 2748 (1995).

[6] J.G.Muga, S.Brouard and D.Macias, Ann.Phys. 240, 351 (1995).

[7] N.Grot, C.Rovelli and R.S.Tate, Phys.Rev. A54, 4676 (1996).

[8] V.Delgado and J.G.Muga, Phys.Rev. A56, 3425 (1997).

[9] V.Delgado, Phys.Rev. A57, 762 (1998).

[10] J.G.Muga, R.Sala Mayato and I.L.Egusquiza (eds), Time in Quantum Mechanics (Springer, Berlin, 2002); J.G.Muga and C.R.Leavens, Phys.Rep. 338, 353 (2000).

[11] J.J.Halliwell and J.M.Yearsley, Phys.Rev. A79, 062101 (2009).

[12] J.J.Halliwell and J.M.Yearsley, Phys.Lett. A374, 154 (2009).

[13] J.M.Yearsley, D.A.Downs, J.J.Halliwell and A.K.Hashagen, Phys. Rev. A 84, 022109 (2011).

[14] S.P.Eveson, C.J.Fewster and R.Verch, Ann.Inst. H.Poincaré 6, 1 (2005).

[15] M.Penz, G.Grübl, S.Kreidl and P.Wagner, J.Phys. A39, 423 (2006).

[16] J.G.Muga, J.P.Palao and C.R.Leavens, Phys.Lett. A253, 21 (1999).

[17] M.V.Berry, J.Phys. A43, 415302 (2010).

[18] P.Strange, Eur. J. Phys. 331147 (2012).

[19] J.M.Yearsley, J.J.Halliwell, R.Hartshorn and A.Whitby, Phys. Rev. A 86, 042116 (2012).

[20] M. Palmero, E. Torrontegui, M. Modugno, J. G. Muga, Phys. Rev. A 87, 053618 (2013).

[21] N.Balazs and B.K.Jennings, Phys.Rep.104, 347 (1984); M.Hillery, R.F.O'Connell, M.O.Scully and E.P.Wigner, Phys.Rep. 106, 121 (1984).

[22] P.Loughlin and L.Cohen, J.Mod.Optics 50, 2305 (2003).

[23] W.Gale, E.Guth and G.T.Trammell, Phys.Rev. 165, 1434 (1968). 
[24] Y.Aharonov and L.Vaidman, Phys.Lett. A178, 38, (1993).

[25] M.Daumer, D.Dürr, S.Goldstein and N.Zanghi, J.Stat.Phys. 88, 967 (1997).

[26] D.J.Mason, M.F.Borunda and E.J.Heller, cond-mat/1205.0291 (2012).

[27] S.O’Mullane, Imperial College MSc dissertation (2013).

[28] F. McLafferty and P. Pechukas, Chem. Phys. Lett. 27, 511 (1974); W. Miller, Acc. Chem. Res. 26, 174 (1993); S. Garashchuk and T. Vazhappilly, J Chem Phys 131, 164108 (2009); T. Seideman and W. H. Miller, J. Chem. Phys. 95, 1768 (1991); T. J. Park and J. C. Light, J. Chem. Phys. 88 (1988).

[29] J.G.Muga, private communication 